\title{
Learning and Memory Deficits After Ibotenate Lesions of the Nucleus Basalis: Reversal by Cholinomimetics
}

\author{
H.C. Fibiger
}

\begin{abstract}
The effects of bilateral ibotenic acid-induced lesions of the nucleus basalis magnocellularis (nBM) on the acquisition and retention of several spatial memory tasks were studied in the rat. Maintenance of spatial memory in a food search task was impaired following $\mathrm{nBM}$ lesions. Acquisition of spontaneous alternation and reinforced alternation in a T-maze was also significantly impaired in animals with these lesions. In contrast, the animals with $\mathrm{nBM}$ lesions were not impaired in the acquisition of a position habit in a T-maze. In several of the tasks there was evidence of some learning in the lesioned animals after substantial training, although they were significantly deficient when compared with the controls. Administration of the cholinergic agonists physostigmine sulfate or pilocarpine nitrate prior to behavioral testing resulted in a rapid and significant improvement in the performance of the lesion animals. The ibotenate-induced lesions significantly reduced the activity of choline acetyltransferase (CAT) in the anterior and the posterior neocortex. Hippocampal CAT activity was not changed. The results indicate that the cholinergic projections originating in the $\mathrm{nBM}$ are involved in the learning and memory of spatial tasks. They also suggest that degeneration of cholinergic neurons in the basal forebrain contributes significantly to the learning and memory deficits observed in Alzheimer's disease.
\end{abstract}

RÉSUMÉ: Déficits au niveau de l'apprentissage et de la mémoire à la suite de lésions du nucleus basalis causées par l'iboténate: cet effet est réversible par les cholinomimétiques. Nous avons étudié les effets de lésions bilatérales provoquées par l'acide iboténique au niveau du nucleus basalis magnocellularis (nBM) chez le rat sur l'acquisition et la rétention de plusieurs tâches relevant de la mémoire spatiale. Il y avait atteinte du processus de conservation au niveau de la mémoire spatiale lors d'une tâche impliquant la recherche d'aliments à la suite de lésions du nBM. L'acquisition de l'alternance spontanée et de l'aternance renforcée dans un labyrinthe en T étaient également atteints de façon significative chez les animaux porteurs de ces lésions. Par contre, les animaux avec des lésions du nBM ne présentaient pas de déficit dans l'acquisition d'un habitus quant à la position dans un labyrinthe en T.

Les animaux porteurs de lésions démontraient un certain degré d'apprentissage après un entrainement sérieux à plusieurs des tâches, même s'ils manifestaient un déficit significatif quand ils étaient comparés aux témoins. L'administration de sulfate de physostigmine ou de nitrate de pilocarpine, deux agonistes cholinergiques, avant l'évaluation comportementale, a entrainé une amélioration rapide et significative dans le rendement des animaux porteurs de lésions. L'activité de la choline acétyltransférase (CAT) dans le néocortex antérieur et postérieur était réduite de façon significative par les lésions induites au moyen de l'iboténate. L'activité de la CAT n'était pas modifiée au niveau de l'hippocampe. Ces résultats démontrent que les projections cholinergiques prenant naissance dans le nBM sont impliquées dans l'apprentissage et la mémorisation des tâches spatiales. Ils suggèrent également que la dégénérescence des neurones cholinergiques dans les noyaux du prosencéphale contribue de façon significative aux déficits de l'apprentissage et de la mémoire dans la maladie d'Alzheimer.

Can. J. Neurol. Sci. 1986; 13:498 